\title{
A Novel Traveling-Wave Feed Technique for Circularly Polarized Planar Antennas
}

\author{
Kum Meng Lum, Chanchai Laohapensaeng, and Charles Free
}

\begin{abstract}
A novel, circularly polarized (CP) microstrip antenna array is described, in which the radiating patches are fed via a slot in the ground plane. Design and simulation data are provided for a prototype antenna working at $5 \mathrm{GHz}$. The antenna provides a high quality $\mathrm{CP}$ wave, and the structure is partially suitable for incorporating in multilayer microwave circuits.
\end{abstract}

Index Terms-Circular polarization, microstrip antennas, traveling-wave feed.

\section{INTRODUCTION}

C IRCULAR polarization is a key feature of many existing and developing mobile communication systems. Moreover, many such systems require the antenna to be closely integrated with the other transceiver circuitry. In this paper, we propose a new technique, which is capable of yielding high quality circular polarization, with a feeding system that is particularly suitable for integration in a multilayer circuit. This type of circuit, fabricated either in thick-film or LTCC, is attractive because it can provide a combination of high-performance and low-cost fabrication.

The proposed traveling-wave feed system offers a number of advantages over other rear-fed patch techniques that have been reported in the literature [1]. It does not require a probe through the dielectric layer beneath the patch and it does not require a resonant aperture beneath the patch.

The current work is an extension of the concept of using a slotline to excite a single patch wave, that was previously reported in the literature [2].

In order to establish the validity of the proposed technique, a prototype $5-\mathrm{GHz}$ antenna was designed and tested. Data are presented that compare the measured and simulated performance of this antenna.

\section{Antenna Structure}

Fig. 1 shows the geometry of the proposed CP antenna. The structure is composed of four planar rectangular radiating patches each with dimensions $L_{p}$ and $W_{p}$, where $L_{p}$ is the resonant dimension for the fundamental mode and $W_{p}$ is the optimum width [3] to give good radiation from each patch. Each patch is linearly polarized, but because there is an annular spacing of $\lambda / 4$ between the patches they will be successively excited $90^{\circ}$ out of phase. This will lead to circular polarization. This concept was originally established by Huang [4] using

Manuscript received June 24, 2004; revised September 10, 2004. The review of this letter was arranged by Associate Editor M. Mrozowski.

The authors are with the University of Surrey, Surrey GU2 7XH, U.K. (e-mail: k.lum@eim.surrey.ac.uk).

Digital Object Identifier 10.1109/LMWC.2005.844218

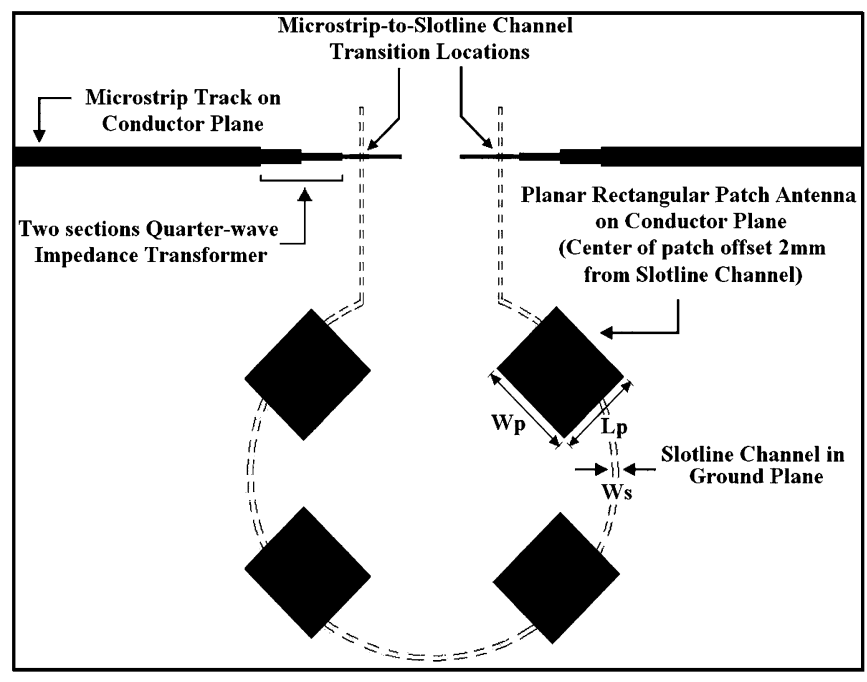

Fig. 1. Geometry of traveling wave feed circularly polarized planar antenna with center of radiating patch offset $2 \mathrm{~mm}$ from slotline channel.

probe fed patches and a corporate feed structure. A circular slotline channel, of width $W_{s}$, runs in the ground plane beneath the patches [5], [6]. The test antenna was fabricated on RT/Duroid 5870 having the following parameters: substrate thickness, $\mathrm{h}=1.575 \mathrm{~mm}$; substrate dielectric constant, $\varepsilon_{r},=2.33 ; 0.5-\mathrm{oz}$ electro-deposited copper. The circuit was fed from $50-\Omega$ microstrip. However, the width of the slotline channel, $W_{S}$, would be very small to give a characteristic impedance of $50 \Omega$ and so, to avoid etching problems, the impedance of the slotline was increased to $110 \Omega$ so that a wider channel could be used. Two, two-section quarter-wave microstrip transformers were used to convert the microstrip line impedance from $50 \Omega$ to $110 \Omega$ [7]. It should be noted that since the slot is still relatively narrow, and the slotline is matched, there would not be significant radiation from the slotline.

Thus the key antenna dimensions were

$$
\begin{aligned}
L_{\mathrm{P}} & =19.000 \mathrm{~mm} \\
W_{\mathrm{P}} & =23.000 \mathrm{~mm} \\
W_{\mathrm{S}} & =00.500 \mathrm{~mm} .
\end{aligned}
$$

The mean diameter of the circular slotline channel was $80.150 \mathrm{~mm}$ so as to give the correct $\lambda / 4$ spacing between adjacent patches. Photographs of the upper and lower surfaces of the antenna are shown in Figs. 2 and 3, respectively.

\section{THEORY}

The slot line was excited through a conventional microstrip-to-slotline transition [8], [9]. A portion of the signal 


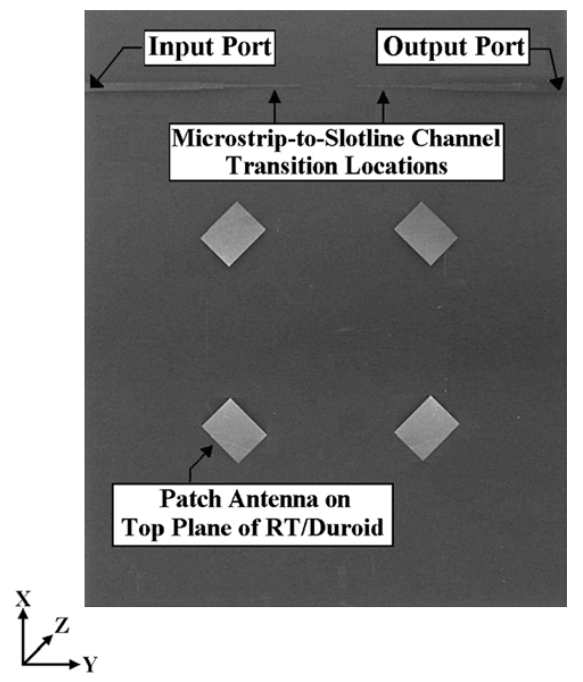

Fig. 2. Photograph of the top surface of the antenna, showing the microstrip feed lines and the radiating patches.

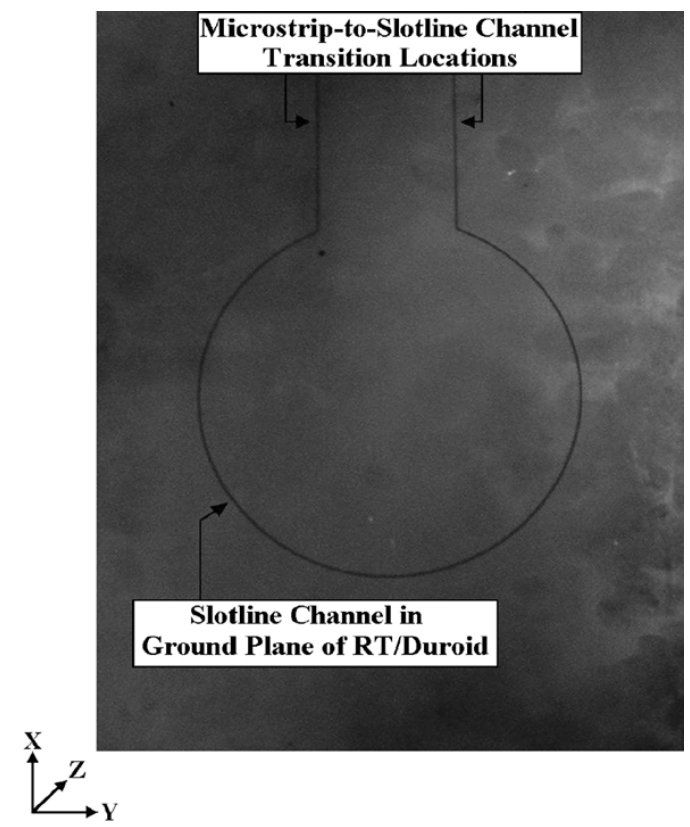

Fig. 3. Photograph of the ground plane of the antenna, showing the slotline channel.

traveling in the slotline will successively excite each patch, providing that the slotline is offset from the center of the patch. The amount of offset will determine the amount of coupling. In this case an offset of $2 \mathrm{~mm}$ was chosen to give minimal coupling to each patch, so that each patch would receive approximately the same signal. In future designs the amount of offset could be varied to compensate for the progressive loss of signal in the slotline.

\section{EXPERIMENTAL RESULTS AND DISCUSSION}

The antenna was simulated using Agilent's ADS software. Measurements were made using an Agilent 8510 Network Analyzer. As shown in Fig. 4, the measured return loss agrees well with the simulated response. It is below $-10 \mathrm{~dB}$ from $4.6 \mathrm{GHz}$ to $5.2 \mathrm{GHz}$.

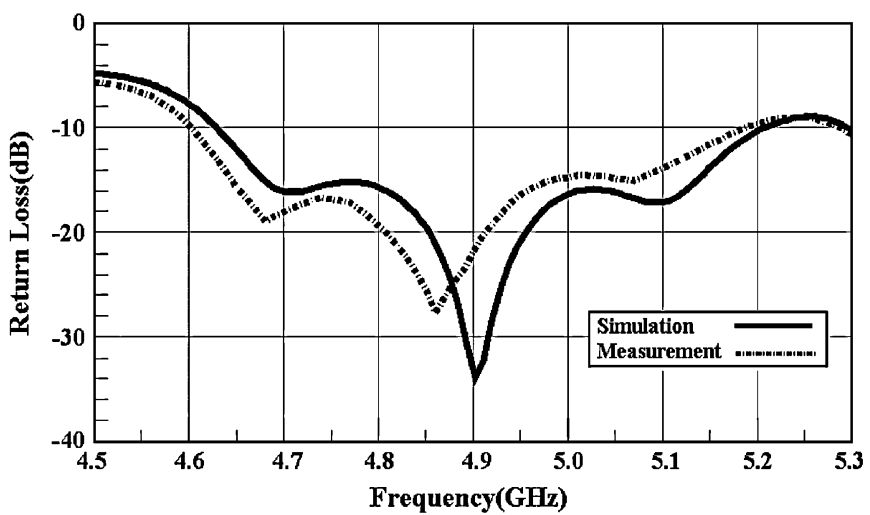

Fig. 4. Return loss of the traveling-wave circularly polarized planar antenna.

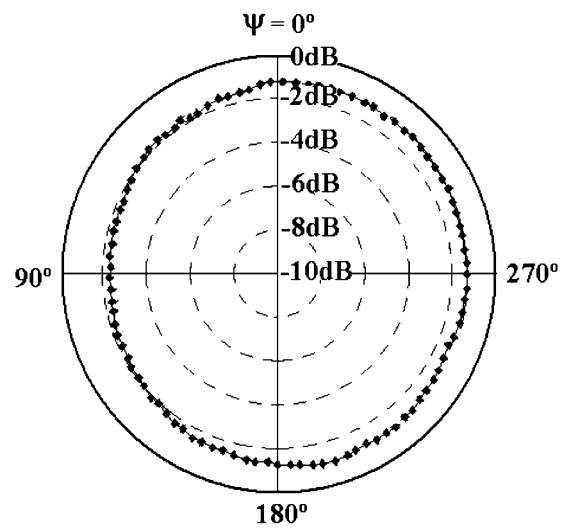

Fig. 5. Measured circular polarization (plot of variation of strength of E-field as a function of angle of rotation at a distance of $1.5 \mathrm{~m}$ from antenna) of the traveling-wave circularly polarized planar antenna. [Referring to Figs. 2 and 3, the polarization plot was taken in the $X Y$-plane, with $Z=1.5 \mathrm{~m}]$.

Fig. 5 shows the measured circular polarization of the antenna array at $4.86 \mathrm{GHz}$. It was found that an adequate combination of good return loss and a good circular polarization existed over a $6 \%$ bandwidth. It is apparent that good circular polarization exists and the key performance of the circuit has been established.

For this prototype antenna, the same slotline offset was used for each patch, and no attempt was made to match the slotline between patches. Some further improvement to the results could be obtained by introducing a variable offset as previously described, together with inter-patch impedance matching in the slotline. Also, efficiency has not been considered in this prototype design, which was intended merely to demonstrate the principle. In future work it is intended to control the amount of energy coupled to each patch so as to minimize the energy dissipated in the impedance terminating the slotline.

\section{CONCLUSION}

The concept of a novel circularly polarized microstrip array using a traveling-wave feed system has been established through practical measurement. High quality circular polarization was demonstrated with very good agreement between the measured and simulated return loss of the antenna. The proposed structure is particularly suitable for inclusion in highly integrated multilayer transceivers. 


\section{REFERENCES}

[1] Handbook of Microstrip Antennas, J. R. James and P. S. Hall, Eds., Peter Peregrinus, London, UK, 1990.

[2] K. P. Tang, C. E. Free, and G. F. Goldspink, "A novel slotline-fed microstrip patch antenna," in Pkroc. IEEE Antennas Propagation Conf., vol. 1, Apr. 1995, pp. 235-238.

[3] C. W. Garvin, R. E. Munson, and L. T. Ostwald, "Missile base mounted microstrip antennas," IEEE Trans. Antennas Propagat., vol. AP-25, no. 9, pp. 604-610, Sep. 1977.

[4] J. Huang, "A Technique for an array to generate circular polarization with linearly polarized elements," IEEE Trans. Antennas Propagat., vol. AP-34, no. 9, pp. 1113-1124, Sep. 1986.
[5] E. A. Mariani, C. P. Heinzman, J. P. Agrios, and S. B. Cohn, "Slot line characteristic," IEEE Trans. Microwave Theory Tech., vol. 17, no. 12, pp. 1091-1096, Dec. 1969.

[6] J. B. Knorr and J. Saenz, "End effect in a shorted slot," IEEE Trans. Microwave Theory Tech., no. 9, pp. 579-580, Sep. 1973.

[7] R. E. Colin, Foundations for Microwave Engineering, 2nd ed. New York: McGraw-Hill, 1992, pp. 356-358.

[8] J. B. Knorr, "Slot-line transitions," IEEE Trans. Microwave Theory Tech., no. 5, pp. 548-554, May 1974.

[9] M. M. Zinieris, R. Sloan, and L. E. Davis, "A broadband microstrip-slotline transition," Microw. Opt. Technol. Lett., vol. 18, no. 5, pp. 339-342, Aug. 1998. 